\title{
A locally adaptive ensemble approach for data-driven prognostics of heterogeneous fleets
}

Proc IMechE Part O: $\int$ Risk and Reliability 2017, Vol. 23I (4) 350-363 (c) IMechE 2017 Reprints and permissions: sagepub.co.uk/journalsPermissions.nav DOI: $10.1177 / 1748006 \times 17693519$ journals.sagepub.com/home/pio (S)AGE

\author{
Sameer Al-Dahidi', Francesco Di Maio', Piero Baraldi' and Enrico Zio ${ }^{1,2}$
}

\begin{abstract}
In this work, we consider the problem of predicting the remaining useful life of a piece of equipment, based on data collected from a heterogeneous fleet working under different operating conditions. When the equipment experiences variable operating conditions, individual data-driven prognostic models are not able to accurately predict the remaining useful life during the entire equipment life. The objective of this work is to develop an ensemble approach of different prognostic models for aggregating their remaining useful life predictions in an adaptive way, for good performance throughout the degradation progression. Two data-driven prognostic models are considered, a homogeneous discretetime finite-state semi-Markov model and a fuzzy similarity-based model. The ensemble approach is based on a locally weighted strategy that aggregates the outcomes of the two prognostic models of the ensemble by assigning to each model a weight and a bias related to its local performance, that is, the accuracy in predicting the remaining useful life of patterns of a validation set similar to the one under study. The proposed approach is applied to a case study regarding a heterogeneous fleet of aluminum electrolytic capacitors used in electric vehicle powertrains. The results have shown that the proposed ensemble approach is able to provide more accurate remaining useful life predictions throughout the entire life of the equipment compared to an alternative ensemble approach and to each individual homogeneous discrete-time finite-state semi-Markov model and fuzzy similarity-based models.
\end{abstract}

\section{Keywords}

Fault prognostics, remaining useful life, locally adaptive ensemble, heterogeneous fleet, homogeneous discrete-time finite-state semi-Markov model, fuzzy similarity-based model, aluminum electrolytic capacitors

\section{Introduction}

In industries such as nuclear, oil and gas, chemical and transportation, unforeseen equipment failures are extremely costly in terms of repair costs, lost revenues, environmental hazards and human fatalities. ${ }^{1}$ To anticipate failures and mitigate their consequences, predictive maintenance approaches are being developed, based on the assessment of the actual equipment degradation condition and on the prediction of its evolution for setting the optimal time for maintenance. ${ }^{1-4}$ The underlying concept is that of failure prognostics, that is, predicting the remaining useful life (RUL) of the equipment undergoing degradation ${ }^{5-8}$ (the amount of time the equipment can continue performing its functions under the operational and working conditions it will experience).

In practice, efficient failure prognostics avoids system failures and unscheduled shutdowns, helps performing efficient maintenance strategies and allows full exploitation of the equipment useful life. Hence, failure prognostics increases the system availability and safety, while reduces maintenance costs. ${ }^{5,6,8-10}$

Approaches for RUL estimation can be generally categorized into model-based and data-driven. 5,6,11-18 Model-based approaches use physics-based models to describe the degradation behavior of the equipment. ${ }^{8,12,19,20}$ For example, Gebraeel and $\operatorname{Pan}^{21}$ presented a degradation modeling framework for RUL prediction of rolling bearings under time-varying

\footnotetext{
'Department of Energy, Politecnico di Milano, Milan, Italy

${ }^{2}$ Chair System Science and the Energy Challenge, Fondation Electricité de France (EDF), CentraleSupélec, Université Paris Saclay, ChatenayMalabry, France
}

\section{Corresponding author:}

Piero Baraldi, Department of Energy, Politecnico di Milano, Via La Masa 34, 20156 Milan, Italy.

Email: piero.baraldi@polimi.it 
operational conditions; Li et al. ${ }^{22,23}$ proposed two prediction models of defect propagation in bearings; Luo et al. ${ }^{17}$ developed a model-based prognostic technique that relies on an accurate simulation model for system degradation prediction and applied the developed technique to a vehicle suspension system. Despite the fact that these approaches have been shown capable of providing accurate prognostic results, the assumptions and simplifications on which they are based may pose limitations on their practical deployment. ${ }^{7,12,24-26}$ On the other side, data-driven prognostic approaches do not use any explicit physics-based model, but rely exclusively on the availability of process data related to equipment health to build (black-box) models that capture the degradation and failure modes of the equipment. $5,8,20,25,27-30$

In this work, the availability of condition monitoring data from similar pieces of equipment, forming what in the industrial context is called a fleet, ${ }^{31,32}$ motivates the development of data-driven prognostic approaches that capitalize on the information contained in such data to estimate the equipment RUL. In practice, heterogeneous fleets of $P$ pieces of equipment, which have different and/or similar technical features, typically undergo different usages under different operating conditions. Thus, even if the fleet data can provide wider knowledge concerning the equipment behavior and, thus, can, in principle, improve the efficiency of the fault prognostics task, ${ }^{31-33}$ they are difficult to be treated within traditional data-driven prognostic schemes.

The main difficulty in prognostics tasks using fleet data is that the equipment typically experiences different operating conditions, which influence both the condition monitoring data and the degradation processes. ${ }^{34}$ Therefore, individual data-driven prognostic models might not provide satisfactory RUL predictions in terms of accuracy: each model can provide accurate RUL predictions under some operating conditions but less accurate in others. ${ }^{35}$ To overcome this, ensemble approaches, based on the aggregation of multiple model outcomes, have been introduced, with superior robustness and accuracy than the individual models ${ }^{36,37}$ and the possibility of estimating the uncertainty of the predictions. ${ }^{38}$

This work proposes an ensemble formed by different data-driven prognostic models, capable of aggregating the RUL predictions in an adaptive way, for good performance throughout the entire degradation trajectory of an equipment.

Two data-driven prognostic base models are considered: (1) a homogeneous discrete-time finite-state semiMarkov model (HDTFSSMM) ${ }^{10,34}$ and (2) a fuzzy similarity-based (FSB) model. ${ }^{24}$ The former approach entails building a statistical model of degradation, estimating its parameters and using the model within a direct Monte Carlo (MC) simulation scheme ${ }^{39}$ to estimate the equipment RUL, whereas the latter model evaluates the similarity between the test degradation trajectory and the available fleet run-to-failure training trajectories and uses the RULs of these latter to estimate the RUL of the former, considering how similar they are. ${ }^{24,40-42}$

The ensemble approach developed tailors the local fusion method developed in Baraldi et al. ${ }^{43}$ to the scope of RUL aggregation. It is based on the following main four steps:

1. Retrieve patterns from the validation set similar to the test pattern under analysis for the prediction. The retrieved validation patterns will be used for optimizing the values of the local fusion method, that is, the weights in Step 2 and the biases in Step 3.

2. Assign a weight to each individual model of the ensemble; the weight is proportional to the model prediction accuracy estimated on the retrieved patterns.

3. Quantify the bias of each individual model of the ensemble; the bias is proportional to the model average RUL prediction error estimated on the retrieved patterns.

4. Aggregate the outputs, accounting for the model's weights and biases.

With respect to Step 1, a novel strategy is proposed for the identification of the patterns of the validation set similar to the test pattern. In Baraldi et al., ${ }^{43}$ the similar patterns are those with the smallest distance from the test pattern under analysis, regardless of the degradation trajectory they belong to. This might cause identifying all the similar patterns in the same degradation trajectory and, thus, the ensemble approach might provide less accurate RUL predictions. This can be justified by the fact that the prediction accuracy of each individual model of the ensemble depends on the diversity and representativeness of the identified patterns that influence the weights assigned to the models. In other words, all degradation trajectories of the validation set can, in principle, bring useful information for determining the RUL of the test trajectory currently developing. Therefore, the proposed strategy considers at most only one similar pattern from each validation trajectory.

With respect to Step 2, three weighting strategies have been considered:

a. Weight proportional to the inverse of the mean absolute error (mae) made by the model on the identified patterns of the validation set similar to the test pattern. ${ }^{43}$

b. Weight proportional to the logarithm of the inverse of the mae. ${ }^{43}$

c. The Borda-count method. ${ }^{36}$

The quantification of the bias of each model in Step 3 consists in calculating the local mean error made by the model on the identified patterns of the validation set similar to the test pattern. ${ }^{43}$ 
With respect to Step 4, the output aggregation is performed by a weighted average of the individual model RUL prediction to which the model local bias (Step 3) is subtracted, with the weights computed in Step 2.

Thus, the original contributions in this work are twofold:

1. The application of the local fusion method ${ }^{43}$ for fault prognostics task.

2. The proposal of a new method for selecting patterns of the validation set most similar to the test pattern.

The proposed approach is applied to a case study regarding a heterogeneous fleet of aluminum electrolytic capacitors used in electric vehicle powertrains. The performance of the proposed approach is verified with respect to the accuracy index (AI) ${ }^{44}$ and is compared with the performance of each individual model. For further comparison, an alternative ensemble approach is applied to the case study and its results are compared to those obtained by the individual models and the proposed ensemble approach. The alternative approach is an adaptive switching ensemble approach for datadriven prognostics that selects the HDTFSSMM at early stages of life and the FSB model at the last stages of life. ${ }^{34,45}$

The remaining of this article is organized as follows. In section "The data-driven prognostic models," the two prognostic models are briefly recalled. In section "The locally adaptive ensemble approach for datadriven prognostics," the proposed ensemble approach for the accurate estimation of the RUL of equipment belonging to a heterogeneous fleet working under variable operating conditions is illustrated. In section "Aluminum electrolytic capacitors in fully electrical vehicles' case study," a case study regarding a heterogeneous fleet of aluminum electrolytic capacitors used in electric vehicle powertrains is described, and the results obtained with the proposed ensemble approach are discussed and compared with each individual model and an alternative adaptive switching ensemble approach. Finally, some conclusions are drawn in section "Conclusion."

\section{The data-driven prognostic models}

This section briefly illustrates the two data-driven prognostic models considered: the HDTFSSMM (section "The HDTFSSMM") proposed by Al-Dahidi et al. ${ }^{10,34}$ and the FSB model (section "The FSB model"), ${ }^{24}$ respectively.

Let us assume that we have available $I_{p}$ measurements for each one of the $p=1, \ldots, P$ pieces of equipment of a heterogeneous fleet monitored at predefined times $t_{1}, t_{2}, \ldots, t_{l}, t_{I_{p}}, l=1, \ldots, I_{p}$. The time interval $t_{l}-t_{l-1}$ between two measurements is assumed to be formed by $M$ discrete time steps. The $P$ pieces of equipment are divided into $P_{\text {train }}$ training, $P_{\text {valid }}$ validation and $P_{\text {test }}$ test sets for the purpose of building the individual models, developing the proposed ensemble approach and verifying its performance, respectively. Each $p$ th trajectory is a $Z$-dimensional trajectory, where $Z$ is the number of signals representative of the equipment behavior and of the operating conditions that the equipment is subjected to. Among the training trajectories, $P_{\text {train }}^{c}$ are complete run-to-failure trajectories (i.e. trajectories that last all the way to the instance when the degradation state reaches the threshold value beyond which the equipment loses its functionality) and $P_{\text {train }}^{i c}=P_{\text {train }}-P_{\text {train }}^{c}$ are incomplete run-to-failure trajectories (i.e. trajectories that do not reach the failure threshold).

\section{The HDTFSSMM}

The degradation process is assumed to follow a homogeneous (i.e. memoryless), discrete-time (i.e. transitions among states occur at discrete time instants), finite-state (i.e. a finite set of degradation states) and semi-Markov (i.e. transition rates depend on the current state sojourn time with any arbitrary distribution) model. ${ }^{46-49}$ The transition rates are taken as discrete Weibull distributions, as these are the probability distributions most commonly used to describe degradation processes of industrial equipment. ${ }^{10,48,50}$

The flowchart for the method is sketched in Figure 1. The method goes along the following two phases: a training phase for building the degradation model and estimating its parameters and a test phase for using the model within a direct MC simulation scheme to estimate the RUL of an equipment. Overall, it entails three main steps: ${ }^{10,34}$

Step 1: Setting up the number of states of the HDTFSSMM. The multidimensional segments of measurements taken from the $P_{\text {train }}$ degradation trajectories are appended in the matrix $\overline{\bar{X}}$. The objective is to partition the collected data in $\overline{\bar{X}}$ into $G$ dissimilar groups (whose number is a priori unknown), such that data belonging to the same group characterize the degradation states of the HDTFSSMM that has to be built.

To this aim, an unsupervised ensemble clustering approach is adopted (refer to Al-Dahidi et al. ${ }^{51}$ and Baraldi et al. ${ }^{52,53}$ for more details): two base clusterings are first performed on two groups of signals (the first populated by signals representative of the equipment behavior and the second representative of the operating conditions) and, then, ensembled to get the final consensus clusters $G$ that can be seen as the states representative of the different degradation levels of the equipment that are influenced and explained by different operating conditions. ${ }^{51}$ The failure state (i.e. an absorbing state) at which the degradation level reaches the failure threshold value is added to those states to build the transition diagram of the equipment operation with $G_{\text {final }}$ states (i.e. $G_{\text {final }}=G+1$ states). 


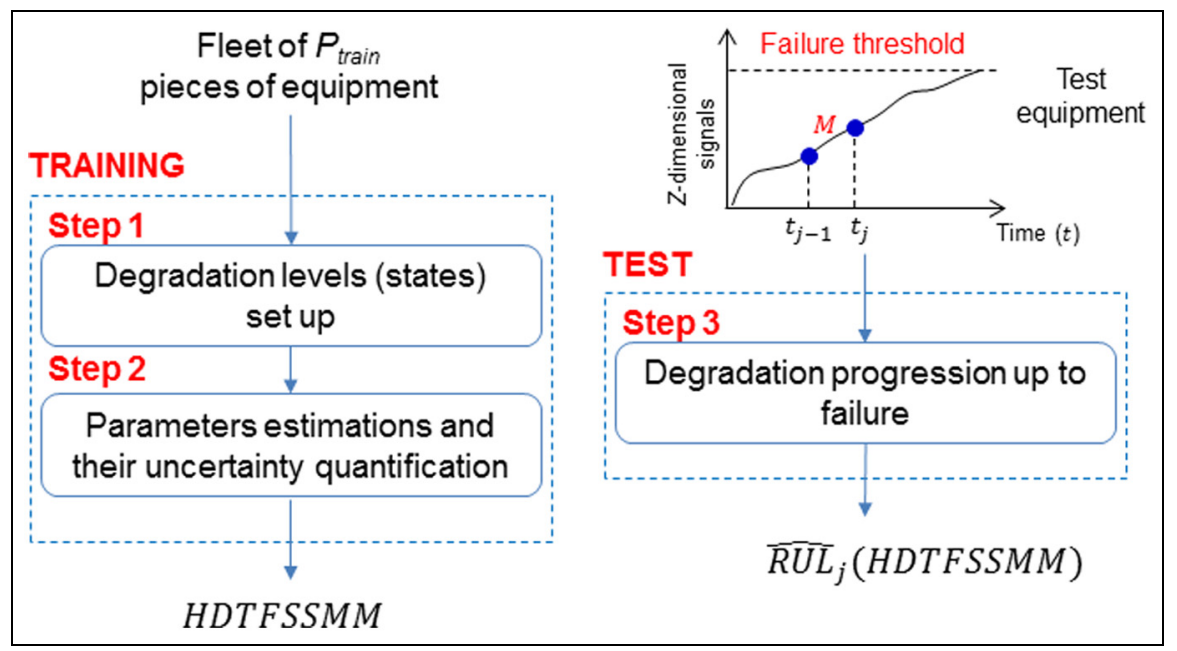

Figure I. Flowchart of the HDTFSSMM.

Phase 2: States transition parameters estimation and their uncertainty quantification. Once the topology of the model is fully defined, the parameters governing the transitions among the degradation states and their uncertainty are to be estimated by resorting to the maximum likelihood estimation (MLE) technique and the Fisher information matrix (FIM), respectively (refer to Kendall and Stuart ${ }^{54}$ for more details).

Phase 3: Direct MC simulation of the degradation progression for the online estimation of the RUL. At the current time $t_{j}$, the RUL provided by the HDTFSSMM $\widehat{R U L}_{j}(H D T F S S M M)$ of a test equipment is estimated using the $M$ latest measurements of the $Z$-dimensional signals and by resorting to the direct MC simulation with $N_{\max }$ trials. ${ }^{55}$

\section{The FSB model}

The idea underpinning this model is to evaluate the similarity between the test trajectory and the $P_{\text {train }}^{c}$ complete run-to-failure reference trajectories available and to use the RULs of these latter to estimate the RUL of the former, considering how similar they are. ${ }^{24}$

The flowchart for the method is sketched in Figure 2. It entails four steps:

Step 1: Pointwise difference computation. At the current time $t_{j}$, the distance $\delta_{l}^{p_{\text {train }}}$ between the sequence of the $M$ latest measurements of the $Z$ signals $\bar{r}_{j-M+1: j}$ of the test trajectory and all $M$-long segments $\bar{r}_{l-M+1: l}^{p_{\text {train }}}, l=1, \ldots, I_{p_{\text {train }}}$ of all reference trajectories $p_{\text {train }}=1, \ldots, P_{\text {train }}$ is computed

$$
\delta_{l}^{p_{\text {train }}}=\sum_{i=1}^{M}\left|\bar{r}_{j-M+i}-\bar{r}_{l-M+i}^{p_{\text {train }}}\right|^{2}
$$

where $|\bar{x}-\bar{y}|^{2}$ is the square Euclidean distance between vectors $\bar{x}$ and $\bar{y}$.

Step 2: Pointwise similarity computation. The similarity $S_{l}^{p_{\text {train }}}$ of the training trajectory segment $\bar{r}_{l-M+1: l}^{p_{\text {train }}}$ to the test segment is defined as a function of the distance measure $\delta_{l}^{p_{\text {train }}}$. In $\mathrm{Di}$ Maio and $\mathrm{Zio},{ }^{24}$ the following bell-shaped function has turned out to give robust results in FSB due to its gradual smoothness ${ }^{24,41,42}$

$$
S_{l}^{p_{\text {train }}}=e^{-\left(\frac{-\ln (\alpha)}{\beta^{2}} \delta_{l}^{p_{\text {train }} 2}\right)}
$$

The arbitrary parameters $\alpha$ and $\beta$ can be set by the analyst to shape the desired interpretation of similarity into the fuzzy set: the larger the value of the ratio $(-\ln (\alpha)) /\left(\beta^{2}\right)$, the narrower the fuzzy set and the stronger the definition of similarity. The choice of the values of $\alpha$ and $\beta$ depends on the application and are typically optimized by trial and error using the trajectories of the validation set. ${ }^{24}$

Step 3: Weight definition. To assign the weight $v^{p_{\text {train }}}$ given to the $p_{\text {train }}$ th reference trajectory accounting for how similar it is to the test segment, the maximum similarity along the $p_{\text {train }}$ th row of the matrix of equation (2) is first identified

$$
S_{l^{*}}^{p_{\text {train }}}=\max _{l=1, \ldots, I_{\text {prain }}} S_{l}^{p_{\text {train }}}
$$

The weight $v^{p_{\text {train }}}$ is, then, computed resorting to the arbitrarily chosen decreasing monotone function, which guarantees that the smaller the minimum distance (the larger the similarity), the larger the weight given to the $p_{\text {train }}$ th reference pattern, where $p_{\text {train }}=1, \ldots, P_{\text {train }}$

$$
v^{p_{\text {train }}}=S_{l^{*}}^{p_{\text {train }}} e^{\left(\frac{1}{\beta}\left(1-S_{l^{*}}^{p_{\text {train }}}\right)\right)}
$$

Then, the weight $v^{p_{\text {train }}}$ is normalized

$$
v^{p_{\text {train }}}=\frac{v^{p_{\text {train }}}}{\sum_{p_{\text {train }}=1}^{P_{\text {train }}} v^{p_{\text {train }}}}
$$

For the prediction of the test equipment RUL, an RUL value $\widehat{r u l}_{l^{*}}^{p_{\text {train }}}$ is assigned to each training 


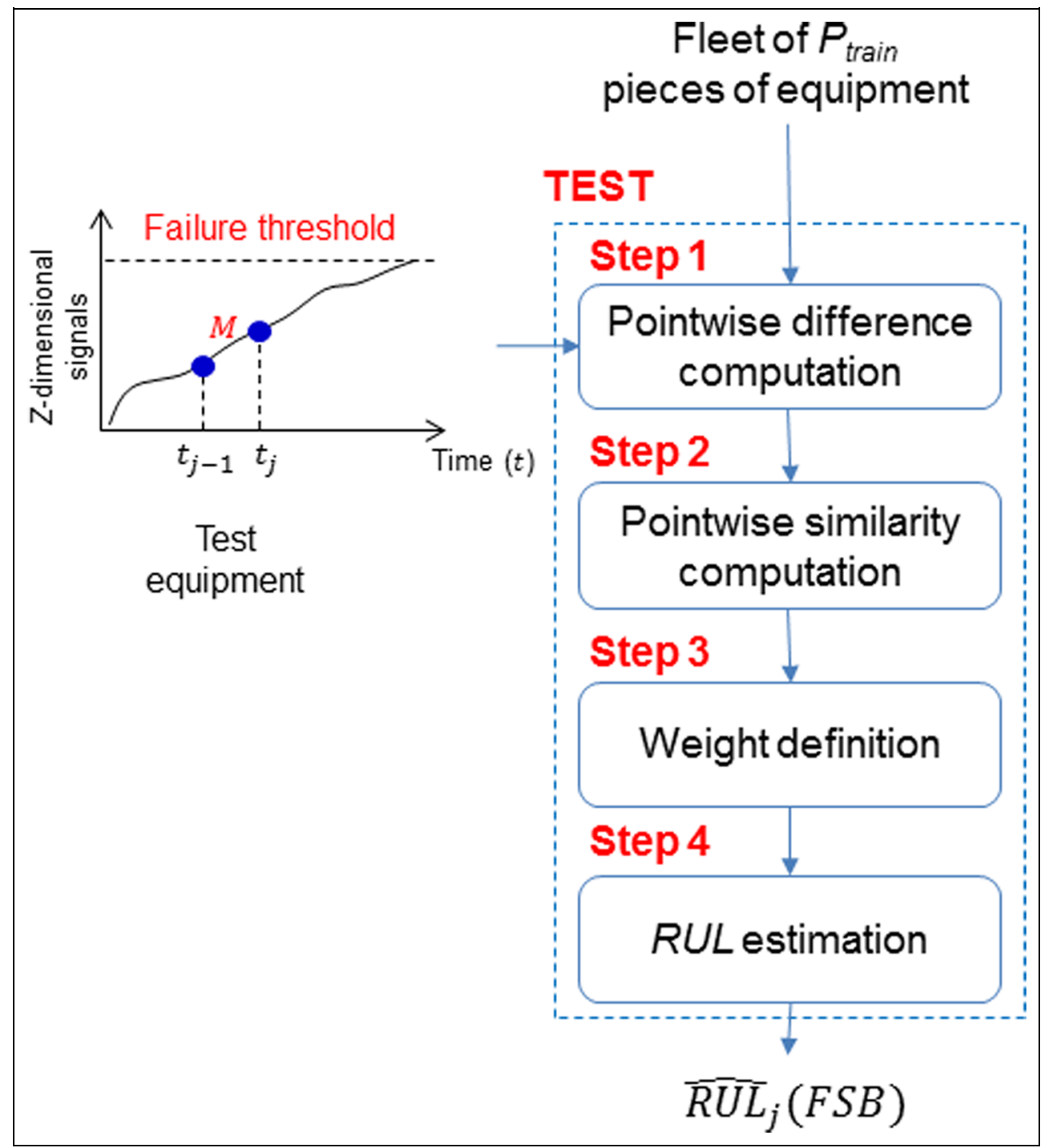

Figure 2. Flowchart of the FSB model.

trajectory $p_{\text {train }}=1, \ldots, P_{\text {train }}$ by considering the difference between the trajectory failure time $t_{F}$ and the last time instant $t_{l^{*}}$ of the trajectory segment $\bar{r}_{l^{*}-M+1: l^{*}}^{p_{\text {tran }}}$, which has the maximum similarity $S_{*^{*}}^{p_{\text {train }}}$ with the test trajectory

$$
\widehat{r u l} l_{l^{*}}^{p_{\text {train }}}=t_{F}-t_{l^{*}}
$$

Step 4: RUL estimation. The RUL prediction of the test equipment at the current time $t_{j}, \widehat{R U L}_{j}(F S B)$, is given by the similarity-weighted sum of the values $\widehat{r u l}_{l^{*}}^{p_{\text {train }}}$

$$
\widehat{R U L}_{j}(F S B)=\sum_{p_{\text {train }}=1}^{P_{\text {train }}} v^{p_{\text {train }}} \widehat{\operatorname{rul}}_{l^{*}}^{p_{\text {train }}}
$$

\section{The locally adaptive ensemble approach for data-driven prognostics}

Let us assume to have available $H$ different prognostic models. We aggregate the RUL predictions for the general test trajectory by dynamically adapting the weights considering the distance of the test pattern to the patterns of a validation set $P_{\text {valid }}$.

More specifically, the aggregation of the prognostic models outcomes requires to associate a weight $w_{j}^{h}$ and a bias $b_{j}^{h}$ to the RUL prediction $\widehat{R U L}_{j}(h)$ of each model $h$. The basic idea consists in correcting the values of $\widehat{R U L}_{j}(h)$ by subtracting the estimated bias $b_{j}^{h}$ and weighting the $\widehat{R U L}_{j}(h)$ with $w_{j}^{h}$. $^{43}$ Notice that weights and biases are different at each test time $j$.

The method flowchart is sketched in Figure 3. It entails five main steps:

Step 1: RUL predictions by the different prognostic models. At the current time $t_{j}, H$ RUL predictions $\widehat{R U L}_{j}(h), h=1, \ldots, H$ are provided by the $H$ prognostic models.

Step 2: Pattern pointwise difference computation. The distance $d_{l}^{p_{\text {valid }}}$ between the sequence of the $M$ latest measurements of the $Z$ signals $\bar{r}_{j-M+1: j}$ of the test trajectory and all $M$-long segment $\bar{r}_{l-M+1: l}^{p_{\text {valid }}}$, $l=1, \ldots, I_{p_{\text {valid }}} \quad$ of all reference trajectories $p_{\text {valid }}=1, \ldots, P_{\text {valid }}$ is computed 


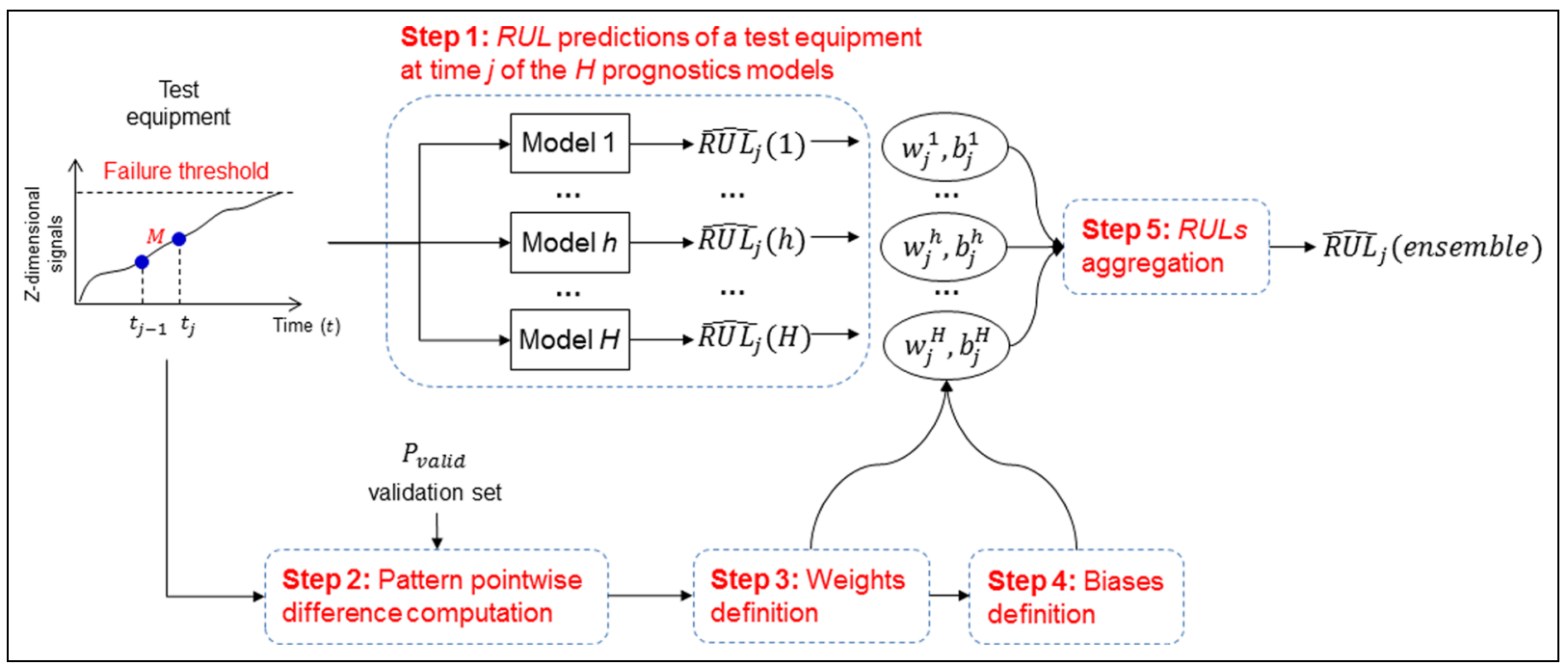

Figure 3. Flowchart of the proposed ensemble approach.

$$
d_{l}^{p_{\text {valid }}}=\sum_{i=1}^{M}\left|\bar{r}_{j-M+i}-\bar{r}_{l-M+i}^{p_{\text {valid }}}\right|^{2}
$$

Step 3: Weight definition. The weight $w_{j}^{h}$ of the $h$ th model is calculated based on its performance in predicting the RUL of the patterns of the validation set which are closer to the test pattern.

In practice, the reference pattern with the minimum distance $d_{l^{*}}^{p_{\text {valid }}}$ is identified for each $p_{\text {valid }}$ th reference trajectory as the pattern with the largest similarity to the test pattern

$$
d_{l^{*}}^{p_{\text {valid }}}=\min _{l=1, \ldots, I_{p_{\text {valid }}}} d_{l}^{p_{\text {valid }}}
$$

Since the local mae $\operatorname{mae}_{j, P_{\text {valid }}}^{h}$ (defined in equation (10)) provides information about the performance of the $h$ th model in predicting the RUL of the $P_{\text {valid }}$ identified patterns, it can be considered an estimation of the error that will affect the RUL prediction of the $h$ th model and thus be used for the calculations of the weight $w_{j}^{h}$

$$
m a e_{j, P_{\text {valid }}}^{h}=\frac{\sum_{p_{\text {valid }}=1}^{P_{\text {valid }}}\left|\widehat{r u l} l_{l^{*}}^{p_{\text {valid }}}(h)-r u l_{l^{*}}^{p_{\text {valid }}}\right|}{P_{\text {valid }}}
$$

where $\widehat{r u l}_{l^{*}}^{p_{\text {valid }}}(h)$ and $r u l_{l^{*}}^{p_{\text {valid }}}$ are the RUL prediction provided by the $h$ th model for the pattern identified from the $p_{\text {valid }}$ trajectory and its true RUL, respectively.

According to Baraldi et al., ${ }^{43}$ three different weighting strategies have been considered:

a. Weights proportional to the inverse of the mae

$$
w_{j}^{h}=\frac{1}{m a e_{j, P_{\text {valid }}}^{h}}
$$

b. Weights proportional to the logarithm of the inverse of the normalized mae

$$
w_{j}^{h}=\log \left[\frac{\max _{P_{\text {valid }, h}}\left|\widehat{r u l} p_{l^{*}, j}^{p_{\text {valid }}}(h)-r u l_{l^{*}, j}^{p_{\text {valid }}}\right|}{m a e_{j, P_{\text {valid }}}^{h}}\right]
$$

where $\max _{P_{\text {valid, }, h}}\left|\widehat{r u l}{\underline{l^{*}, j}}_{\text {valid }}^{p_{1}}(h)-r u l_{l^{*}, j}^{p_{\text {valid }}}\right|$ is the maximum value of the error over all patterns of the validation set $P_{\text {valid }}$ and all models $h, h=1, \ldots, H$. $^{43}$

c. Weights are assigned according to the Bordacount method. ${ }^{36}$ The estimated local error is used to make a ranking of the different models and to assign them a score $C_{j}^{h}, 1<C_{j}^{h}<H$, according to their position in the ranking, that is, 1 for the worst performing model and $H$ for the best performing one

$w_{j}^{h}=C_{j}^{h}$

Step 4: Bias calculations. The bias correction $b_{j}^{h}$ of the $h$ th model is taken equal to the local mean error

$$
m e_{j, P_{\text {valid }}}^{h}=\frac{\sum_{p_{\text {valid }}=1}^{P_{\text {valid }}}\left(\widehat{r u l} l_{l^{*}}^{p_{\text {valid }}}(h)-r u l_{l^{*}}^{p_{\text {valid }}}\right)}{P_{\text {valid }}}
$$

This quantity represents the accuracy of the RUL predictions obtained by each model $h$ on the $P_{\text {valid }}$ patterns of the validation set closer to the test pattern.

Step 5: Aggregation of the RULs provided by the individual models. Once the weights and the biases are calculated, each $\widehat{R U L}_{j}(h)$ is corrected by subtracting the estimated bias $b_{j}^{h}$ and, then, combined with the others by means of a weighted average ${ }^{43}$ 


$$
\widehat{R U L}_{j}(\text { ensemble })=\frac{\sum_{h=1}^{H} w_{j}^{h} \cdot\left(\widehat{R U L}_{j}(h)-b_{j}^{h}\right)}{\sum_{h=1}^{H} w_{j}^{h}}
$$

\section{Aluminum electrolytic capacitors in fully electrical vehicles' case study}

The potential benefit of using the proposed ensemble approach is demonstrated in a case study regarding a heterogeneous fleet of $P=150$ aluminum electrolytic capacitors used in electric vehicle powertrains. ${ }^{34,56}$ The performance of the proposed approach in providing accurate RUL estimates is here compared with those of each individual model and of an alternative ensemble approach.

\section{The available data}

The main degradation mechanism of electrolytic capacitors is the vaporization of the electrolyte, whose degradation speed is largely influenced by the component working temperature. ${ }^{57}$

During the capacitor life, the following $Z=2$ signals are measured:

1. ESR $R^{\text {measured }}$ is a direct measurement of the component degradation.

2. The temperature $T$ experienced by the capacitor, which represents the operating condition most influencing the degradation process of the capacitor.

Given the unavailability of real data describing the degradation of a fleet of capacitors, the degradation trajectories have been simulated by applying a physicsbased model of the electrolyte vaporization. ${ }^{56,58}$

According to Rigamonti et al., ${ }^{56}$ the normalized equivalent series resistance $E S R^{\text {norm }}$ is considered as a degradation indicator. The physics-based degradation model is represented by a first-order Markov process

$$
E S R_{t}^{\text {norm }}=E S R_{t-1}^{\text {norm }} e^{F\left(T_{t-1}\right)}+\omega_{t-1}
$$

where $\omega_{t-1}$ is the process noise at time $t-1$ and $F\left(T_{t-1}\right)$ is a coefficient which defines the degradation rate of the capacitor depending on the capacitor working temperature at time $t-1$.

The equation linking the measurements to the $E^{\text {norm }}$ is

$$
\operatorname{ESR}_{t}^{\text {measured }}=\operatorname{ESR}_{t}^{\text {norm }} \cdot\left(a+b e^{-\frac{\left(T_{t}^{E S R}-273.15\right)}{c}}\right)+\eta_{t}
$$

where $a, b$ and $c$ are the measurement parameters, $T_{t}^{E S R}$ is the temperature at which the measurement has been performed at time $t$ (usually different from the aging temperature that the capacitor experienced) and $\eta_{t}$ is the measurement noise at time $t .{ }^{59}$
The simulation of the evolution of the $E S R^{\text {norm }}$ for a fleet of capacitors is performed by assuming an initial value equal to $100 \%$ and iteratively applying equation (16) with a time step equal to $1 \mathrm{~h}$. The failure time of the capacitor is defined as the time at which $E S R^{\text {norm }}$ of the capacitor reaches the failure threshold of $200 \%{ }^{58}$

The measured ESR values, ESR ${ }^{\text {measured }}$, have been obtained by applying equation (17) to the numerically simulated degradation indicator values $E S R^{\text {norm }}$ for arbitrary parameter values,${ }^{56}$ and the possible temperature profiles have been simulated by taking into account the suggestions of design experts of the motor behavior: ${ }^{60,61}$ temperature variations experienced by the capacitors during life are mainly caused by (1) the seasonality of the environmental external temperature and (2) the aging (barely up to $10 \%$ of its initial temperature value). Therefore, the simulated temperature profiles follow an arbitrary sinusoidal function that justifies seasonality, by adding to this a shift sigmoidal function accounting for aging.

The heterogeneity among the $P=150$ capacitors that belong to the fleet is guaranteed by considering arbitrary parameter values for the sinusoidal and the sigmoidal functions describing the operating conditions.

For clarification purposes, Figure 4 shows the simulated data of two capacitors (capacitor 1 and capacitor 2-dark and light shade of color, respectively): Figure 4 (top) shows $E S R^{\text {norm }}$, Figure 4 (left bottom) shows $E S R^{\text {measured }}$, whereas Figure 4 (right bottom) shows the $T$ profiles experienced by the capacitors. It is worth noticing that the higher the temperature, the faster the vaporization process due to the increase in the self-heating effects and, hence, the faster the failure process too as shown in Figure 4 (top, capacitor 2light shade of color). ${ }^{56,62}$

The whole data set is divided into $P_{\text {train }}=100$ training, $P_{\text {valid }}=25$ validation and $P_{\text {test }}=25$ test trajectories. Among the $P_{\text {train }}=100$ trajectories, $P_{\text {train }}^{c}=20$ last all the way to the failure threshold, whereas $P_{\text {train }}^{i c}=80$ are incomplete, that is, measurement data are not available until failure. For clarification purposes, Figure 5 shows the $E S R^{\text {norm }}$ of the complete and incomplete run-to-fail degradation trajectories (in dark and light shade of color, respectively).

All the measurements of the $P_{\text {train }}=100$ trajectories are stored in the matrix $\overline{\bar{X}}$ that is used to build the individual models (as presented in section "Implementation of the ensemble approach"), and thus, to develop the ensemble approach. For computational convenience, 1000 time steps between two successive measurements (i.e. $M=1000$ ) are considered.

\section{Implementation of the ensemble approach}

The individual models are built using the trajectories of the $P_{\text {train }}=100$ capacitors. With respect to the HDTFSSMM, the whole set is used to build the degradation model and estimate its parameters, and 


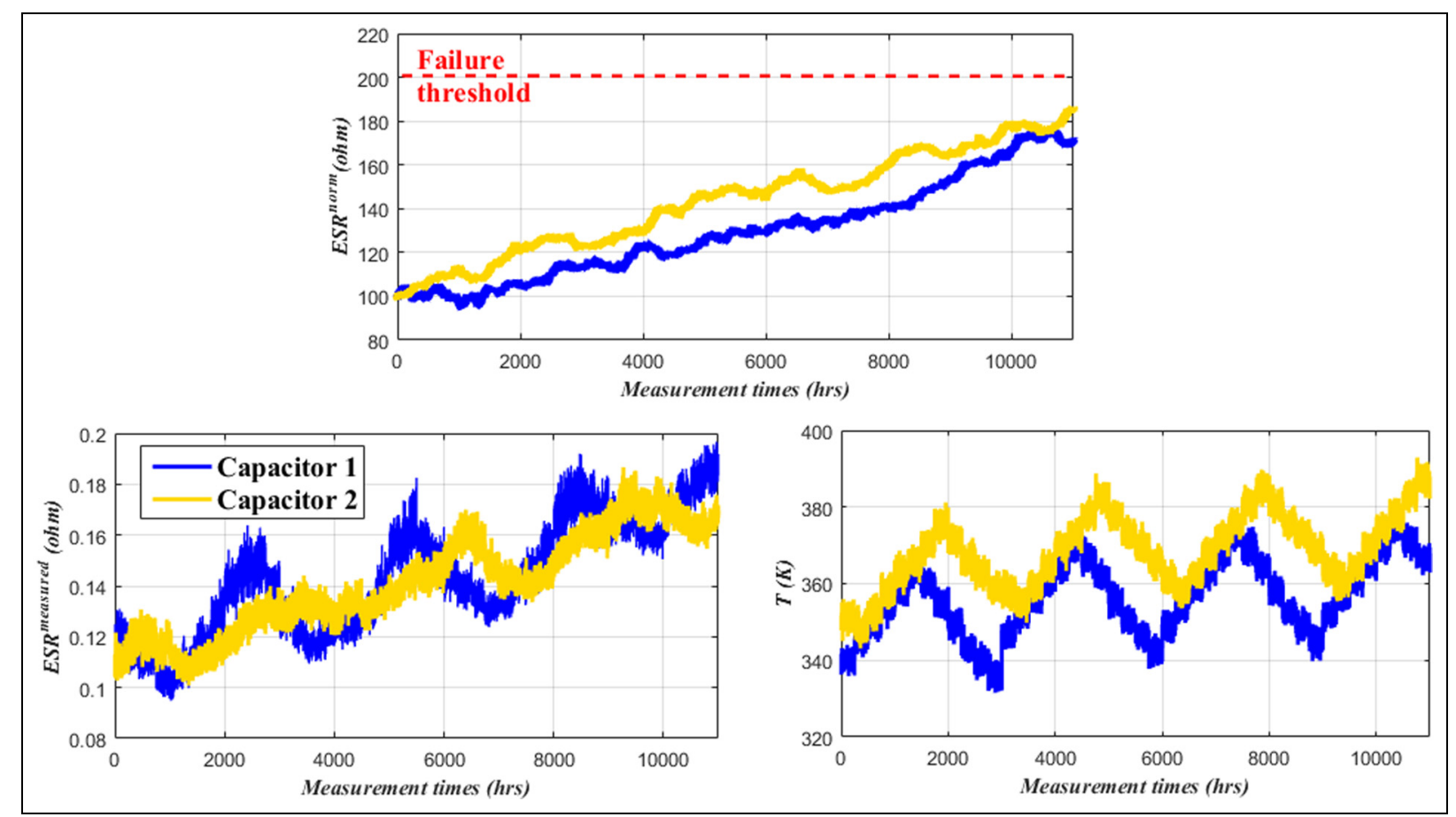

Figure 4. The true degradation process (top), the ESR measurements (left bottom) and the temperature profiles experienced by the capacitors (right bottom).

Table I. Value of the Al for the $P_{\text {test }}=25$ trajectories obtained by the proposed ensemble approach and the individual models.

\begin{tabular}{llll}
\hline & HDTFSSMM & FSB model & Locally adaptive ensemble approach \\
\hline Al & 1.24 & 0.64 & Weight strategy (a): 0.42 \\
& & Weight strategy (b): 0.37 \\
& & Weight strategy (c): 0.47 \\
\hline
\end{tabular}

HDTFSSMM: homogeneous discrete-time finite-state semi-Markov model; FSB: fuzzy similarity-based; Al: accuracy index.

$N_{\max }=1000 \mathrm{MC}$ trials have been used in the direct $\mathrm{MC}$ simulation step aimed at predicting the RUL of the $P_{\text {test }}=25$ capacitors. ${ }^{34}$ With respect to the FSB model, only the $P_{\text {train }}^{c}=20$ complete run-to-failure training trajectories are used to build a reference library for estimating the RUL of the $P_{\text {test }}=25$ capacitors.

Finally, for each $p_{\text {test }}$ th capacitor, $p_{\text {test }}=1, \ldots, P_{\text {test }}$, and at each time $t_{j}, j=1, \ldots, I_{p_{\text {test }}}$, the proposed ensemble approach is applied following the scheme presented in section "The locally adaptive ensemble approach for data-driven prognostics" using $P_{\text {valid }}=25$ capacitors for the purpose of aggregating the outcomes of the individual models.

The evaluation metric considered in this work is the $\mathrm{AI})^{44}$ that is defined as the relative error of the RUL prediction. In practice, small AI values indicate more accurate predictions. The AI evaluation metric is defined by ${ }^{44}$

$$
A I^{p_{\text {test }}}=\sum_{j=1}^{I_{p_{\text {test }}}} \frac{\left|\widehat{r u l}_{j}^{p_{\text {test }}}-r u l_{j}^{p_{\text {test }}}\right|}{r u l_{j}^{p_{\text {test }}}}, \quad A I=\frac{\sum_{p_{\text {test }}=1}^{P_{\text {test }}} A I^{p_{\text {test }}}}{P_{\text {test }}}
$$

where $A I^{p_{\text {test }}}$ and $A I$ are the average AI of the $p_{\text {test }}$ th equipment and of the overall $P_{\text {test }}$ pieces of equipment, respectively.

\section{Results}

Table 1 reports the average values of the AI for the three alternative weight strategies and the individual models. It can be seen that the ensemble approach with any weighting scheme outperforms any of the individual model in terms of the AI and that the ensemble with the weight strategy (b) achieves the most accurate RUL predictions (i.e. smallest AI equal to 0.37), with $42.19 \%$ improvement with respect to the best individual model, FSB, whose AI is 0.64 .

In Figure 6 (top), the RUL estimates obtained by the proposed ensemble approach (weighting strategy (b)) for two capacitors are plotted in solid line, together with those obtained by the HDTFSSMM and the FSB in circles and squares, respectively.

The analysis of Figure 6 suggests that 


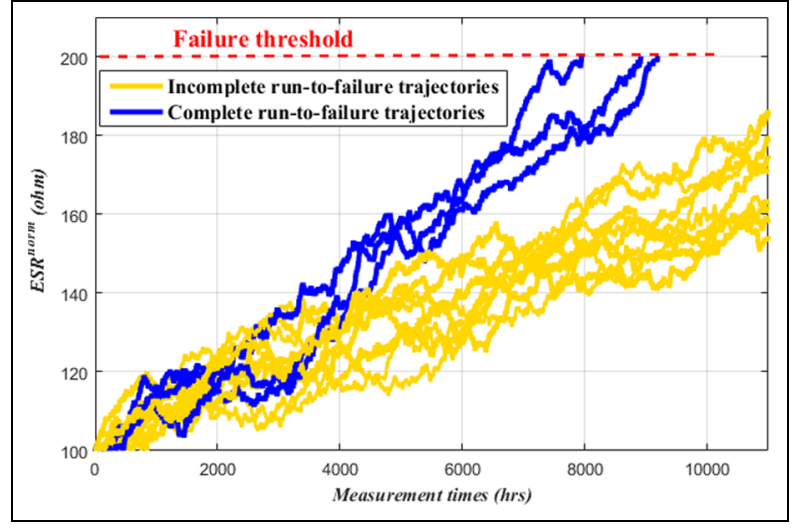

Figure 5. Examples of simulated complete and incomplete run-to-failure degradation trajectories.

1. The predictions provided by the two models are comparable: even if the HDTFSSMM provides more accurate RUL predictions at the early stages of the capacitor life, the FSB model provides more accurate predictions when the capacitor approaches the end of life.

2. The ensemble of the two models, instead, allows obtaining more accurate predictions throughout the lives of the capacitors than each individual model.

Figure 6 (bottom) shows the weights dynamically assigned to the two models at each time $t$ :

1. The HDTFSSMM gets a larger weight from the beginning of the lives to approximately $t=12,500 \mathrm{~h}$ compared with the FSB model. This can be justified by the fact that the HDTFSSMM exploits information taken from both the complete and the incomplete run-to-failure trajectories, whereas the FSB model only uses the first source of information. Furthermore, the complete run-tofailure trajectories used for training the FSB model are characterized by short lives (see Figure 5) and, thus, the FSB model tends, on average, to underestimate the capacitor RUL at the beginning of its degradation trajectory.

2. The FSB model gets exceptionally large weights toward the end of the capacitors' lives compared with the HDTFSSMM. This can be justified by the fact that the HDTFSSMM based on a statistical model for the estimation of the Weibull distributed transition time is not effective when the capacitors approach the failure times.

On the basis of this considerations, one might argue that an alternative approach that uses only the HDTFSSMM for the early stage of the capacitor life and, then, only the FSB model might be superior (from the methodological point of view) and more efficient. The following section "Comparison with the adaptive switching ensemble approach" compares the performances of the proposed ensemble approach of section "The locally adaptive ensemble approach for datadriven prognostics" with that of this latter alternative, developed as in Al-Dahidi et al. ${ }^{45}$

\section{Comparison with the adaptive switching ensemble approach}

The approach is structured into two phases: ${ }^{45}$ an offline selection of the optimal switching time $t_{\text {opt }}$ before which
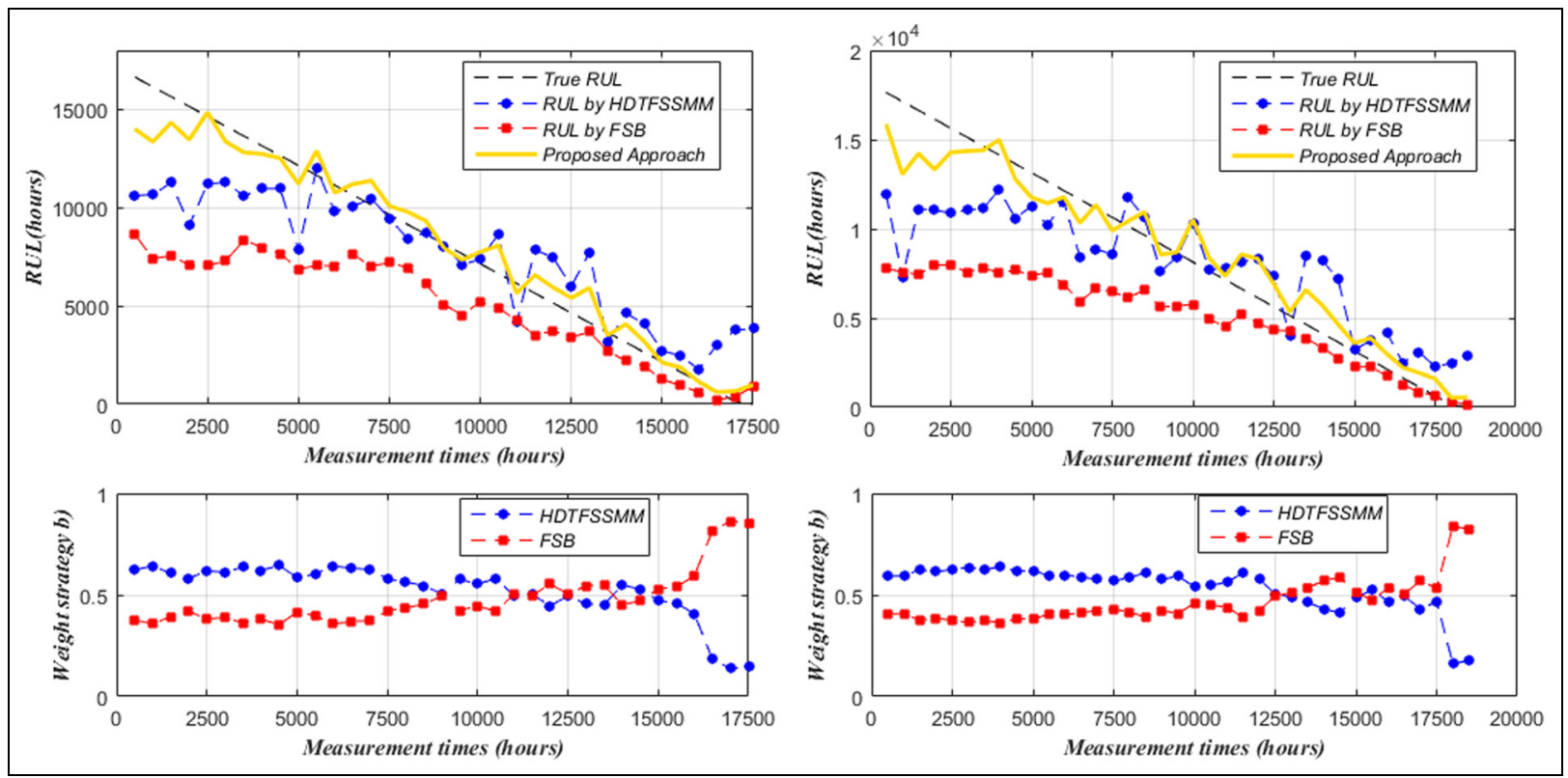

Figure 6. Comparison of the RUL predictions for two capacitors provided by the proposed ensemble approach and each individual model of HDTFSSMM and FSB. 


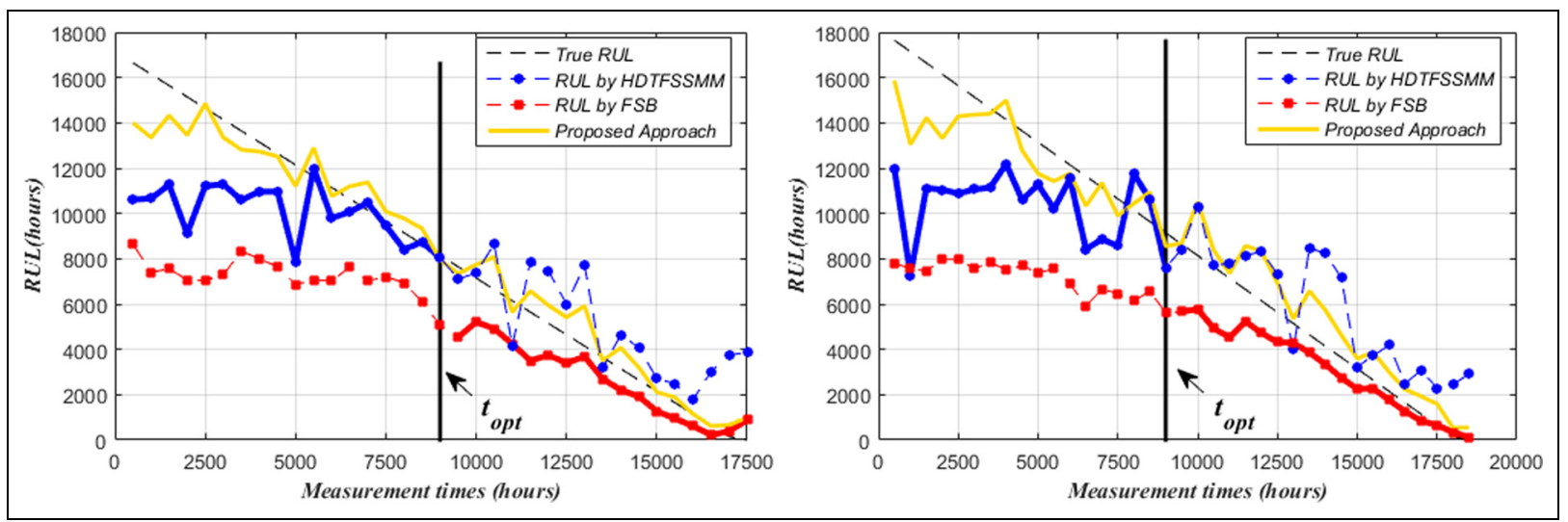

Figure 7. Comparison of the RUL predictions for two capacitors provided by the proposed ensemble approach, the switching ensemble approach and each single model of HDTFSSMM and FSB.

Table 2. Values of the Al for the $P_{\text {test }}=25$ test trajectories.

\begin{tabular}{lll}
\hline & Locally adaptive ensemble approach (weighting strategy $(b))$ & Adaptive switching ensemble approach \\
\hline $\mathrm{Al}$ & 0.37 & $0.5 \mathrm{I}$ \\
\hline
\end{tabular}

Al: accuracy index.

the HDTFSSMM is used for providing the RUL estimates and after which the FSB is used (the interested reader may refer to Appendix 2 for further details on the procedure) and an online phase that relies on $t_{\text {opt }}$ to switch between the HDTFSSMM and the FSB for predicting the RUL of the $P_{\text {test }}=25$ capacitors.

For the case of interest, by adopting a trial-and-error procedure using the validation set trajectory, $t_{\text {opt }}$ turns out to be equal to $9000 \mathrm{~h}$.

Table 2 reports the AI calculated on the $P_{\text {test }}=25$ test trajectories for the locally adaptive ensemble approach (weighting strategy (b)) compared with the adaptive switching ensemble approach. Notice that the proposed ensemble approach is more satisfactory, since it provides lower AI values.

The estimates of the RUL obtained by the adaptive switching ensemble approach for two capacitors are shown in Figure 7 in dark solid lines before and after $t_{\text {opt }}$ (together with those obtained by the locally adaptive ensemble approach in light solid line and each individual model in circle and square markers). It can be easily noticed that the proposed ensemble approach outperforms the adaptive switching ensemble approach in terms of accuracy throughout the entire lives of the capacitors.

\section{Conclusion}

The operating conditions experienced during the life of an equipment influence both the condition monitoring data and the degradation processes. Thus, prognostics for a heterogeneous fleet of equipment working under variable operating conditions is a complex and difficult task, and prognostic approaches based on the use of individual data-driven models might not provide satisfactory predictions of the RUL in terms of accuracy throughout the entire life of the equipment.

In this work, we have proposed an ensemble approach based on the use of two data-driven prognostic models: an HDTFSSMM and an FSB model. The RUL predictions provided by the two models are aggregated using a locally weighted strategy which assigns a weight and a bias using a measure of a local performance of the ensemble individual models, that is, the accuracy in predicting the RUL of patterns of a validation set similar to the one under study.

The proposed approach is capable of (1) benefiting from the availability of condition monitoring data collected from heterogeneous fleets and (2) aggregating the RUL predictions in an adaptive way, for good performance throughout the entire degradation trajectory of an equipment and, thus, enhancing the RUL estimation.

Thus, the main original contributions of this work are as follows:

1. The application of the local fusion method developed in Baraldi et al. ${ }^{43}$ for fault prognostics task.

2. The proposal of a new method for selecting patterns of the validation set most similar to the test pattern.

The proposed approach has been applied to a case study regarding a heterogeneous fleet of aluminum electrolytic capacitors used in electric vehicle powertrains. The performance of the proposed approach has been compared with the performance of each individual model and to an alternative ensemble approach, showing its feasibility and benefit when dealing with data collected from heterogeneous fleets.

Future work will be devoted to (1) the comparison of the proposed ensemble approach to model-based 
prognostic approaches and (2) the application of the proposed ensemble approach on real industrial degradation trajectories collected from the operations of a fleet of industrial equipment.

\section{Declaration of Conflicting Interests}

The author(s) declared no potential conflicts of interest with respect to the research, authorship and/or publication of this article.

\section{Funding}

The author(s) disclosed receipt of the following financial support for the research, authorship, and/or publication of this article: The participation of E.Z. to this research was partially supported by the China NSFC under grant number 71231001 .

\section{References}

1. Baraldi P, Mangili F and Zio E. A belief function theory based approach to combining different representation of uncertainty in prognostics. Inf $S c i(N y)$ 2015; 303: 134-149.

2. McCall JJ. Maintenance policies for stochastically failing equipment: a survey. Manage Sci 1965; 11(5): 493-524.

3. Wei Z, Tao T, ZhuoShu D, et al. A dynamic particle filter-support vector regression method for reliability prediction. Reliab Eng Syst Saf 2013; 119: 109-116.

4. Zhao Z, Wang F-L, Jia M-X, et al. Predictive maintenance policy based on process data. Chemom Intell Lab Syst 2010; 103(2): 137-143.

5. Zio E. Prognostics and health management of industrial equipment. In: Diagnostics and prognostics of engineering systems: methods and techniques. IGI-Global, 2012, pp.333-356. DOI: 10.4018/978-1-4666-2095-7.ch017.

6. Tobon-Mejia D, Medjaher K, Zerhouni N, et al. Hidden Markov models for failure diagnostic and prognostic. In: Prognostics and system health management conference, PHM'11, Shenzhen, China, 24-25 May 2011, pp.1-8. New York: IEEE.

7. Jardine AKS, Lin D and Banjevic D. A review on machinery diagnostics and prognostics implementing condition-based maintenance. Mech Syst Signal Process 2006; 20(7): 1483-1510.

8. Heng A, Zhang S, Tan ACC, et al. Rotating machinery prognostics: state of the art, challenges and opportunities. Mech Syst Signal Process 2009; 23(3): 724-739.

9. Bevilacqua $\mathbf{M}$ and Braglia M. Analytic hierarchy process applied to maintenance strategy selection. Reliab Eng Syst Saf 2000; 70(1): 71-83.

10. Al-Dahidi S, Di Maio F, Baraldi P, et al. Supporting maintenance decision with empirical models based on fleet-wide data. In: The 49th ESReDA seminar on: innovation through human factors in risk assessment \& maintenance, Bruxelles, Belgium, 29-30 October 2015, pp.1-12. European Safety, Reliability and Data Association (ESReDA).

11. Sankavaram C, Pattipati B, Kodali A, et al. Model-based and data-driven prognosis of automotive and electronic systems. In: 2009 IEEE international conference on automation science and engineering (CASE 2009), Bangalore, India, 22-25 August 2009, pp.96-101. IEEE.
12. Chiang LH, Russell E and Braatz RD. Fault detection and diagnosis in industrial systems. 2001. Springer Science \& Business Media.

13. Schwabacher $\mathrm{M}$ and Goebel K. A survey of artificial intelligence for prognostics. In: Proceedings of 2007 AAAI Fall Symposium: AI for Prognostics, Arlington, VA, USA, 9-11 November 2007, pp.107-114. Association for the Advancement of Artificial Intelligence (AAAI).

14. Si XS, Wang W, Hu $\mathrm{CH}$, et al. Remaining useful life estimation - a review on the statistical data driven approaches. Eur J Oper Res 2011; 213(1): 1-14.

15. Sikorska JZ, Hodkiewicz M and Ma L. Prognostic modelling options for remaining useful life estimation by industry. Mech Syst Signal Process 2011; 25(5): 18031836.

16. Lee J, Wu F, Zhao W, et al. Prognostics and health management design for rotary machinery systems-reviews, methodology and applications. Mech Syst Signal Process 2014; 42(1-2): 314-334.

17. Luo S, Pattipati J, Qiao KR, et al. Model-based prognostic techniques applied to a suspension system. IEEE Trans Syst Man Cybern: Part A 2008; 38(5): 1156-1168.

18. Gebraeel J, Elwany N and Pan A. Residual life predictions in the absence of prior degradation knowledge. IEEE Trans Reliab 2009; 58(1): 106-117.

19. Liu J, Wang W, Ma F, et al. A data-model-fusion prognostic framework for dynamic system state forecasting. Eng Appl Artif Intell 2012; 25(4): 814-823.

20. Zio E and Di Maio F. Fatigue crack growth estimation by relevance vector machine. Expert Syst Appl 2012; 39(12): 10681-10692.

21. Gebraeel J and Pan N. Prognostic degradation models for computing and updating residual life distributions in a time-varying environment. IEEE Trans Reliab 2008; 57(4): 539-550.

22. Li Y, Kurfess TR and Liang SY. Stochastic prognostics for rolling element bearings. Mech Syst Signal Process 2000; 14(5): 747-762.

23. Li Y, Billington $\mathrm{S}$, Zhang $\mathrm{C}$, et al. Adaptive prognostics for rolling element bearing condition. Mech Syst Signal Process 1999; 13(1): 103-113.

24. Di Maio F and Zio E. Failure prognostics by a datadriven similarity-based approach. Int J Reliab Qual Saf Eng 2014; 20(1): 1350001

25. Tran VT and Yang B-S. Machine fault diagnosis and prognosis: the state of the art. Int J Fluid Mach Syst 2009; 2(1): 61-71.

26. Li W and Pham H. An inspection-maintenance model for systems with multiple competing processes. IEEE Trans Reliab 2005; 54(2): 318-327.

27. Schwabacher M. A survey of data-driven prognostics. In: Proceedings of the AIAA infotech@aerospace conference, Arlington, VA, 26-29 September 2005, pp.1-5. The American Institute of Aeronautics and Astronautics (AIAA).

28. Coble JB and Hines JW. Prognostic algorithm categorization with PHM challenge application. In: 2008 international conference on prognostics and health management, PHM 2008, Denver, CO, USA, 6-9 October 2008. New York: IEEE.

29. Heimes FO. Recurrent neural networks for remaining useful life estimation. In: International conference on prognostics and health management. PHM 2008, Denver, CO, USA, 6-9 October 2008, pp.1-6. New York: IEEE. 
30. Di Maio F, Tsui KL and Zio E. Combining relevance vector machines and exponential regression for bearing residual life estimation. Mech Syst Signal Process 2012; 31: 405-427.

31. Medina-Oliva G, Voisin A, Monnin M, et al. Predictive diagnosis based on a fleet-wide ontology approach. Knowledge-Based Syst 2014; 68: 40-57.

32. Monnin M, Abichou B, Voisin A, et al. Fleet historical cases for predictive maintenance. In: International conference on acoustical and vibratory methods in surveillance and diagnostics, Surveillance 6, Compiègne, France, 25-26 October 2011. Sciencesconf.org.

33. Agarwal V, Lybeck NJ, Bickford R, et al. Development of asset fault signatures for prognostic and health management in the nuclear industry. In: IEEE conference on prognostics and health management (PHM), Cheney, WA, USA, 22-25 June 2014. IEEE.

34. Al-Dahidi S, Di Maio F, Baraldi P, et al. Remaining useful life estimation in heterogeneous fleets working under variable operating conditions. Reliab Eng Syst Saf 2016; 156: 109-124.

35. $\mathrm{Hu} \mathrm{C}$, Youn BD and Wang P. Ensemble of data-driven prognostic algorithms with weight optimization and $\mathrm{k}$-fold cross validation. In: Annual conference of the prognostics and health management (PHM) society, Portland, Oregon, 10-16 October 2010, pp. 1-12.

36. Polikar R. Ensemble based systems in decision making. IEEE Circuits Syst Mag 2006; 6(3): 21-45.

37. Di Maio F, Hu J, Tse P, et al. Ensemble-approaches for clustering health status of oil sand pumps. Expert Syst Appl 2012; 39(5): 4847-4859.

38. Baraldi $\mathrm{P}$, Mangili $\mathrm{F}$ and Zio E. Investigation of uncertainty treatment capability of model-based and datadriven prognostic methods using simulated data. Reliab Eng Syst Saf 2013; 112: 94-108.

39. Cadini F, Zio E and Avram D. Monte Carlo-based filtering for fatigue crack growth estimation. Probabilistic Eng Mech 2009; 24(3): 367-373.

40. Wang T, Yu J, Siegel D, et al. A similarity-based prognostics approach for Remaining Useful Life estimation of engineered systems. In: International conference on prognostics and health management, Denver, CO, USA, 6-9 October 2008, pp.1-6. IEEE.

41. Zio E and Di Maio F. A data-driven fuzzy approach for predicting the remaining useful life in dynamic failure scenarios of a nuclear system. Reliab Eng Syst Saf 2010; 95(1): 49-57.

42. Angstenberger L. Dynamic fuzzy pattern recognition with applications to finance and engineering. In: International series in intelligent technologies, 2001, vol. 17. The Netherlands: Springer.

43. Baraldi P, Cammi A, Mangili F, et al. Local fusion of an ensemble of models for the reconstruction of faulty signals. IEEE Trans Nucl Sci 2010; 57(2): 793-806.

44. Saxena A, Celaya J, Saha B, et al. Metrics for offline evaluation of prognostic performance. Int J Progn Heal Manag 2010; 1: 1-20.

45. Al-Dahidi S, Di Maio F, Baraldi P, et al. A switching ensemble approach for remaining useful life estimation of electrolytic capacitors. In: Safety and reliability: Methodology and applications - Proceedings of the 26th European safety and reliability conference, ESREL 2016, Glasgow, UK, 25-29 September 2016, pp. 2000-2005.
46. Dong $M$ and Peng $Y$. Equipment PHM using nonstationary segmental hidden semi-Markov model. Robot Comput Integr Manuf 2011; 27: 581-590.

47. Chen CT. Dynamic preventive maintenance strategy for an aging and deteriorating production system. Expert Syst Appl 2011; 38(5): 6287-6293.

48. Moghaddass R and Zuo MJ. A parameter estimation method for a condition-monitored device under multistate deterioration. Reliab Eng Syst Saf 2012; 106: 94-103.

49. Shu MH, Hsu BM and Kapur KC. Dynamic performance measures for tools with multi-state wear processes and their applications for tool design and selection. Int $J$ Prod Res 2010; 48(16): 4725-4744.

50. Moghaddass R and Zuo MJ. An integrated framework for online diagnostic and prognostic health monitoring using a multistate deterioration process. Reliab Eng Syst Saf 2014; 124: 92-104.

51. Al-Dahidi S, Di Maio F, Baraldi P, et al. A novel ensemble clustering for operational transients classification with application to a nuclear power plant turbine. Int J Progn Heal Manag 2015; 6(SP3): 1-21.

52. Baraldi P, Di Maio F, Rigamonti M, et al. Unsupervised clustering of vibration signals for identifying anomalous conditions in a nuclear turbine. J Intell Fuzzy Syst 2013; 28(4): 1723-1731.

53. Baraldi P, Di Maio F and Zio E. Unsupervised clustering for fault diagnosis in nuclear power plant components. Int J Comput Intell Syst 2013; 6(4): 764-777.

54. Kendall $\mathrm{M}$ and Stuart A. The advanced theory of statistics, vol. 2. London: Charles Griffin and Company Limited, 1968.

55. Compare M, Baraldi P, Cannarile F, et al. Homogeneous finite-time, finite-state, semi-Markov modelling for enhancing Empirical Classification System diagnostics of industrial components (under review). Probabilistic Eng Mech 2015.

56. Rigamonti M, Baraldi P, Zio E, et al. Particle filter-based prognostics for an electrolytic capacitor working in variable operating conditions. IEEE Trans Power Electron 2016; 31(2): 1567-1575.

57. Kulkarni C, Biswas G, Koutsoukos X, et al. Integrated diagnostic/prognostic experimental setup for capacitor degradation and health monitoring. in AUTOTESTCON (proceedings), Orlando, FL, 13-16 September 2010, pp.351-357. New York: IEEE.

58. Venet P, Darnand H and Grellet G. Detection of faults of filter capacitors in a converter. Application to predictive maintenance. 15th Int Telecommun Energy Conf INTELEC'93 1993; 2: 229-234.

59. Abdennadher K, Venet P, Rojat G, et al. A real-time predictive-maintenance system of aluminum electrolytic capacitors used in uninterrupted power supplies. IEEE T Ind Appl 2010; 46(4): 1644-1652.

60. Lahyani A, Venet P, Grellet $G$, et al. Failure prediction of electrolytic capacitors during operation of a switchmode power supply. IEEE T Power Electr 1998; 13(6): 1199-1207.

61. Gasperi ML. Life prediction model for aluminum electrolytic capacitors. IEEE Ind Appl Conf 1996 Thirty-First IAS Annu Meet IAS '96, Conf Rec 1996; 3(1): 1347-1351.

62. Wolfgang E. Examples for failures in power electronics systems. In: ECPE tutorial on reliability of power electronic systems, Nuremberg, Germany, 19-20 April 2007, ECPE European Center for Power Electronics e.V. 
Appendix I

\section{Notation}

$a, b, c$

AI

$A I^{p_{\text {test }}}$

$b_{j}^{h}$

$d_{l}^{p_{\text {valid }}}$

$d_{l^{*}}^{p_{\text {valid }}}$

ESR ${ }^{\text {norm }}$

F

$g$

G

$G_{\text {final }}$

$h$

$H$

$I_{p}$

$I_{p_{\text {test }}}$

$I_{p_{\text {train }}}$

$I_{p_{\text {valid }}}$

$K N N$

$l$

$m a e_{j, P_{\text {valid }}}^{h}$

$m e_{j, P_{\text {valid }}}^{h}$

$M$

$N_{\max }$

$p$

$p_{\text {test }}$

$p_{\text {train }}$ parameter characteristics of the capacitor

average accuracy index of the

$P_{\text {test }}$ pieces of equipment

average accuracy index of the $p_{\text {test }}$

equipment

bias associated with the $h$ th

prognostic model at time $t_{j}$

pointwise difference between

$\bar{r}_{j-M+i}$ and $\bar{r}_{l-M+i}^{p_{\text {valid }}}$

minimum distance $d_{l}^{p_{\text {valid }}}$ of $p_{\text {valid }}$

trajectory at time $t_{l^{*}}$

capacitor degradation indicator

coefficient which defines the

degradation rate of the capacitor index of degradation state,

$g=1, \ldots, G_{\text {final }}$

number of degradation states

(final consensus clusters) of

equipment

number of degradation states

including the failure state of

equipment

index of the prognostic model,

$h=1, \ldots, H$

number of individual prognostic

models

number of measurements of $p$ th

equipment

number of measurements of $p_{\text {test }}$

equipment

number of measurements of $p_{\text {train }}$

equipment

number of measurements of $p_{\text {valid }}$

equipment

$\mathrm{K}$-nearest neighbors

index of the measurement time,

$l=1, \ldots, I_{p}$

local mean absolute error

obtained by the $h$ th prognostic

model at time $t_{j}$ of the $P_{\text {valid }}$

trajectories

local mean error obtained by the

$h$ th prognostic model at time $t_{j}$ of

the $P_{\text {valid }}$ trajectories

number of discrete time steps

between two successive

measurements, $t_{l}-t_{l-1}$

number of MC simulation trials

index of equipment in the fleet,

$p=1, \ldots, P$

index of equipment used for

testing, $p_{\text {test }}=1, \ldots, P_{\text {test }}$

index of equipment used for

training, $p_{\text {train }}=1, \ldots, P_{\text {train }}$ $p_{\text {valid }}$

$P$

$P_{\text {test }}$

$P_{\text {train }}$

$P_{\text {train }}^{c}$

$P_{\text {train }}^{i c}$

$P_{\text {valid }}$

$Q_{j}^{h}$

$r u l_{j}^{p_{\text {test }}}$

$r u l_{l^{*}}^{p_{\text {train }}}$

$r u l_{l^{*}}^{p_{\text {valid }}}$

$\bar{r}_{j-M+1: j}$

$\bar{r}_{l-M+1: l}^{p_{\text {train }}}$

$\bar{r}_{l-M+1: l}^{p_{\text {valid }}}$

$\widehat{r u l}_{j}^{p_{\text {test }}}$

$\widehat{r u l}_{l^{*}}^{p_{\text {valid }}}(h)$

$\widehat{R U L}_{j}($ ensemble $)$

$\widehat{R U L}_{j}(F S B)$

$\widehat{R U L}_{j}$

(HDTFSSMM)

$S_{l}^{p_{\text {train }}}$

$S_{l^{*}}^{p_{\text {train }}}$

$t_{F}$

$t_{j}$

$t_{l}$ index of equipment used for

validation, $p_{\text {valid }}=1, \ldots, P_{\text {valid }}$

number of pieces of equipment in the fleet

number of pieces of equipment in the fleet used for testing

number of pieces of equipment in the fleet used for training

number of complete-run-to-

failure equipment used for

training

number of incomplete-run-to-

failure equipment used for

training

number of pieces of equipment in the fleet used for validation

score provided to the $h$ th

prognostic model in the Borda-

count method at time $t_{j}$

true RUL of the $p_{\text {test }}$ capacitor at time $t_{j}$

true RUL of the $p_{\text {train }}$ trajectory at time $t_{l^{*}}$

true RUL of the $p_{\text {valid }}$ trajectory

at time $t_{l^{*}}$

$j$ th segment of length $M$ of a test trajectory

lth segment of length $M$ of $p_{\text {train }}$ reference trajectory,

$l=1, \ldots, I_{p_{\text {train }}, p_{\text {train }}=1, \ldots,}$

$P_{\text {train }}$

$l$ th segment of length $M$ of $p_{\text {valid }}$

trajectory, $l=1, \ldots, I_{p_{\text {valid }}}$,

$p_{\text {valid }}=1, \ldots, P_{\text {valid }}$

RUL prediction of the $p_{\text {test }}$

capacitor at time $t_{j}$

RUL prediction provided by the $h$ th prognostic model for a $p_{\text {valid }}$ trajectory at time $t_{l^{*}}$

ensemble RUL prediction of the

$H$ prognostic models at time $t_{j}$ of a test trajectory

RUL prediction provided by the

FSB model for a test trajectory at time $t_{j}$

RUL prediction provided by the

HDTFSSMM for a test

equipment at time $t_{j}$

measure of similarity between

$\bar{r}_{l-M+1: l}^{p_{\text {train }}}$ and $\bar{r}_{j-M+1: j}$

largest similarity between the

$p_{\text {train }}$ trajectory and the $j$ th

segment of a test trajectory at

time $t_{l^{*}}$

failure time of the $p_{\text {train }}$ trajectory,

$p_{\text {train }}=1, \ldots, P_{\text {train }}$

$j$ th test time of a test equipment

lth measurement time of an

equipment 


\begin{tabular}{|c|c|}
\hline$t_{l^{*}}$ & $\begin{array}{l}\text { last time instant of the segment } \\
\bar{r}_{l^{*}-M}^{p_{\text {train }}}+1: l^{*} \text { which has the } \\
\text { maximum similarity with the test } \\
\text { trajectory }\end{array}$ \\
\hline$t_{\text {opt }}$ & $\begin{array}{l}\text { optimum switching time of the } \\
\text { adaptive switching ensemble } \\
\text { approach }\end{array}$ \\
\hline$t_{s w}$ & $\begin{array}{l}\text { possible switching time of the } \\
\text { adaptive switching ensemble } \\
\text { approach, } t_{s w}=\left[t_{s w}^{\min }, t_{s w}^{\max }\right]\end{array}$ \\
\hline$T_{t}$ & $\begin{array}{l}\text { aging temperature experienced by } \\
\text { the capacitor at time } t\end{array}$ \\
\hline$T_{t}^{E S R}$ & $\begin{array}{l}\text { capacitor temperature at which } \\
\text { the } E S R \text { measurement has been } \\
\text { performed at time } t\end{array}$ \\
\hline$v^{p_{\text {train }}}$ & $\begin{array}{l}\text { weight assigned to the } p_{\text {train }} \\
\text { reference trajectory in the FSB } \\
\text { model, } p_{\text {train }}=1, \ldots, P_{\text {train }}\end{array}$ \\
\hline$w_{j}^{h}$ & $\begin{array}{l}\text { weight associated with the } h \text { th } \\
\text { prognostic model at time } t_{j}\end{array}$ \\
\hline$\overline{\bar{X}}$ & $\begin{array}{l}\text { dataset matrix of the collected } \\
\text { measurements }\end{array}$ \\
\hline$z$ & index of signal \\
\hline$Z$ & $\begin{array}{l}\text { number of signals of each } \\
\text { degradation trajectory }\end{array}$ \\
\hline$\alpha, \beta$ & $\begin{array}{l}\text { parameters of the bell-shaped } \\
\text { similarity function of the FSB } \\
\text { model }\end{array}$ \\
\hline
\end{tabular}

$\delta_{l}^{p_{\text {train }}}$

$\eta_{t}$

$\omega_{t}$

\section{Appendix 2}

\section{The adaptive switching ensemble approach}

The adaptive switching ensemble mode ${ }^{45}$ (sketched in Figure 8) entails first an offline selection of the optimal switching time $t_{\text {opt }}$ among all the possible switching times $t_{s w}=\left[t_{s w}^{\min }, t_{s w}^{\max }\right]$, where $t_{s w}^{\min }$. is the first measurement time and $t_{s w}^{m a x}$ is the longest end of life that minimizes the accuracy index (AI) over the $P_{\text {valid }}$ validation trajectories, that is, the relative error of the RUL prediction. ${ }^{44}$

Then, an online usage for predicting the RUL of $P_{\text {test }}$ pieces of equipment. In other words, the optimal switching time $t_{\text {opt }}$ represents the time up to which HDTFSSMM is used for providing the RUL estimates at the early stage of the equipment life and beyond which FSB is used when the equipment approaches the end of life.

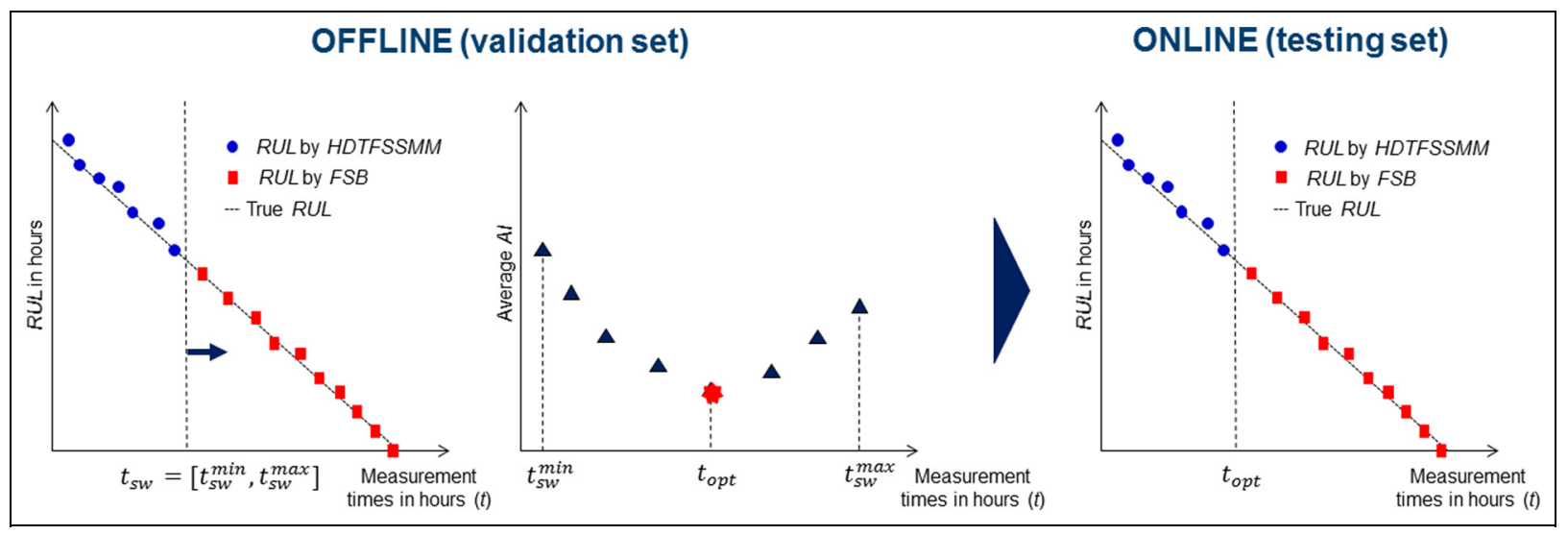

Figure 8. Flowchart of the adaptive switching ensemble approach.. 